\title{
Direct Laser Interference Patterning: Theory and Application
}

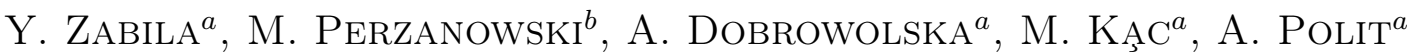 \\ AND M. MARSZAEEK ${ }^{a}$ \\ ${ }^{a}$ The H. Niewodniczański Institute of Nuclear Physics, Polish Academy of Sciences \\ Radzikowskiego 152, 31-342 Kraków, Poland \\ ${ }^{b}$ University of Science and Technology, al. Mickiewicza 30, 30-054 Kraków, Poland
}

\begin{abstract}
We simulated and experimentally investigated the formation of periodic structures generated by multibeam interference patterning. The simulations at the different setup geometry show that resulting interference pattern is quasi-periodical. The calculated patterns show that the symmetries of the interference maxima depend mostly on the angles of incidence and that a wide variety of patterns can be obtained. Because of the difficulty in aligning four beams sufficiently well to avoid secondary periodicities, for testing we used a three-beam interference configuration. Atomic force microscopy images showed good correspondence between the experimental and simulated interference image, with flat islands which correspond to the destructive interference and narrow channels which correspond to the constructive interference fringes.
\end{abstract}

PACS numbers: 68.55.--a, 68.65.Ac

\section{Introduction}

In recent years periodic nanostructures have attracted great interest because of their potential applications in different fields like nanostructured optical devices, nanosieves, nanochannels for fluids, and high-density magnetic recording. In particular, in the field of information storage further increase in storage densities in the presence of instability of recorded data against thermal decay, requires the use of nanoparticle media. In this case in process of recording information is stored in a single nanoparticle [1] with uniaxial magnetic anisotropy, and the direction of magnetization at remanence $[2,3]$ represent bit of information. Different approaches to fabrication of patterned media have been proposed, besides other techniques like focused ion beam etching and e-beam lithography also direct laser interference patterning (DLIL) is significantly developed $[4,5]$. It allows the production of very precise nanometer sized structures, uniformly, and over large areas.

The interference pattern produced by two, three or four beams of a high-power pulsed laser allows a direct, periodically local heating of metallic surfaces through the local photo-thermal interaction between laser and metal. In the process of light interaction with metals, the light quanta are absorbed by conducting electrons, which dissipate the absorbed energy to thermal lattice vibrations. This process occurs within a layer of thickness about $10^{-8} \mathrm{~m}$, corresponding to the penetration depth of light in metal [6].

The method permits the creation of periodic pattern of features with a well-defined long-range order on metallic surfaces. The topography and structure of material surfaces strongly influence their properties and even their functions; therefore a well-defined surface structure, e.g. spatially modulated chemical composition, crystallinity, topography, or structure, will give the surface a completely new property. The main advantage of laser interference patterning is that it requires only single technological procedure in contrast to other methods. Besides that it does not need the masks and relatively large area can be patterned.

This paper presents the calculations of laser light intensity coming from interference of 2,3 and 4 coherent light beams which can be used for direct laser interference patterning.

We have calculated the influence of the optical system geometry on periodicity, linearity and contrast of interference images. These calculations allow us to choose the optimal configuration demanded for carrying out the laser patterning. The optical system for laser patterning based on Nd:YAG laser was designed and mounted. For comparison of theoretical simulations with experiment the structure of the $\mathrm{Si} /(\mathrm{Co}(1 \mathrm{~nm}) / \mathrm{Cu}(2 \mathrm{~nm}))_{3}$ thin films have been modified by laser interference irradiation.

\section{Theoretical simulations}

Figure 1 shows the geometry used to carry out the simulations. In case of two light beams linear interference pattern is obtained (shown in Fig. 2a) with an intensity distribution given by

$$
\frac{I}{I_{0}}=4 \cos ^{2}\left(\frac{\pi\left(r_{1}-r_{2}\right)}{\lambda}\right)
$$




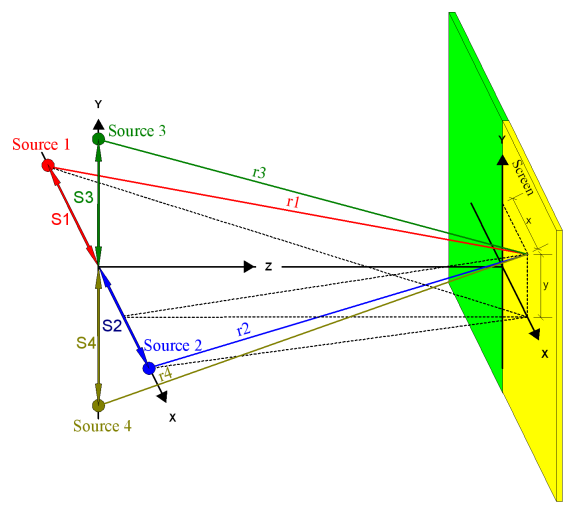

Fig. 1. The schematic view of the geometry used for simulations of interference patterns. S1, S2, S3, S4 are the distances between the reflection of laser beam from positioning mirrors with respect to middle of symmetry $0, z$ - distance between sample plane and mirror plane.

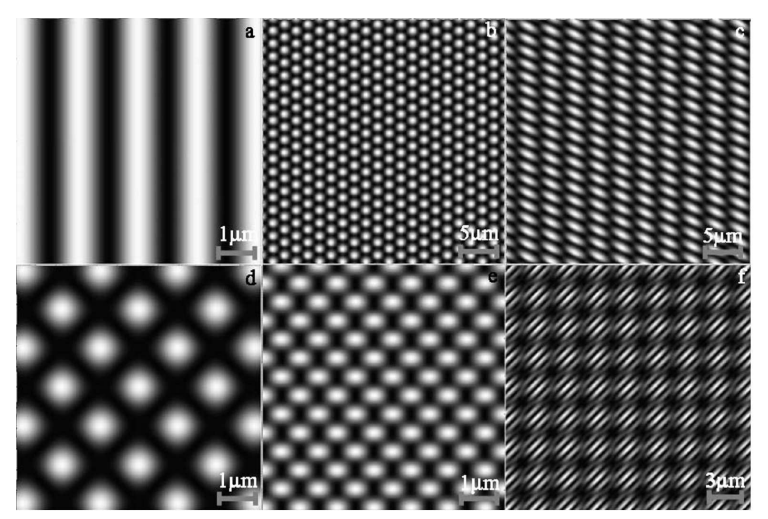

Fig. 2. The interference patterns calculated for the geometry indicated in Fig. 1 for $\lambda=1024 \mathrm{~nm}$ and 2 laser beams (a) $\mathrm{S} 1=\mathrm{S} 2=0.025 \mathrm{~m}$, 3 laser beams (b) $\mathrm{S} 1=\mathrm{S} 2=0.025 \mathrm{~m}$, $\mathrm{S} 3=0.06 \mathrm{~m}$, (c) $\mathrm{S} 1=\mathrm{S} 2=0.025 \mathrm{~m}, \mathrm{~S} 3=0.06 \mathrm{~m}$, the sample declined at $\pi / 3$ with respect to $x y$ plane, and 4 laser beams (d) $\mathrm{S} 1=\mathrm{S} 2=\mathrm{S} 3=\mathrm{S} 4=0.04 \mathrm{~m}$, (e) $\mathrm{S} 1=$ $\mathrm{S} 2=\mathrm{S} 3=\mathrm{S} 4=0.5 \mathrm{~m}$, (f) $\mathrm{S} 1=\mathrm{S} 3=0.06 \mathrm{~m}, \mathrm{~S} 2=\mathrm{S} 4$ $=0.6 \mathrm{~m}$. In all cases $z=0.06 \mathrm{~m}$.

where $I$ - total intensity of interfering beams with individual intensity $I_{0}$.

The intensity distribution in the case of 3 and 4 beams is determined by more complicated formulae

$$
\begin{aligned}
\frac{I}{I_{0}} & =4\left[\cos ^{2}\left(\frac{\pi\left(r_{1}-r_{2}\right)}{\lambda}\right)+\cos ^{2}\left(\frac{\pi\left(r_{1}-r_{3}\right)}{\lambda}\right)\right. \\
& \left.+\cos ^{2}\left(\frac{\pi\left(r_{2}-r_{3}\right)}{\lambda}\right)\right]-3
\end{aligned}
$$

for 3 beams, and

$$
\begin{aligned}
& \frac{I}{I_{0}}=4\left[\cos ^{2}\left(\frac{\pi\left(r_{1}-r_{2}\right)}{\lambda}\right)+\cos ^{2}\left(\frac{\pi\left(r_{1}-r_{3}\right)}{\lambda}\right)\right. \\
& +\cos ^{2}\left(\frac{\pi\left(r_{1}-r_{4}\right)}{\lambda}\right)+\cos ^{2}\left(\frac{\pi\left(r_{2}-r_{3}\right)}{\lambda}\right)
\end{aligned}
$$

$$
\left.+\cos ^{2}\left(\frac{\pi\left(r_{2}-r_{4}\right)}{\lambda}\right)+\cos ^{2}\left(\frac{\pi\left(r_{3}-r_{4}\right)}{\lambda}\right)\right]-8
$$

for 4 beams.

The maxima of these relations $\left(I / I_{0}\right)_{\max }=4,9$ and 16 for 2, 3 and 4 beams, correspondingly, with respect to the intensity of laser beam obtained after splitting, while the minima $\left(I / I_{0}\right)_{\min }=0$ are beam number independent. In all simulations presented in this section, the four beams are assumed to have $700 \mathrm{~nm}$ wavelength and the same electric field amplitudes.

More complex structures demonstrated in Fig. 2b-f can be obtained using 3 and 4 laser beams. The round, ellipsoidal, oval and striped dots can be patterned by 3 or 4 beams depending on the relative positions of laser beams and angle of their incidence on the interference plane.

\section{Experimental details}

For laser interference patterning we have designed an original setup, shown schematically in Fig. 3, mounted from commercially available optical elements. A high power pulsed Nd:YAG laser with a wavelength of $1064 \mathrm{~nm}$, and pulse duration of $10 \mathrm{~ns}$ was used for the laser interference patterning. The energy of single pulse

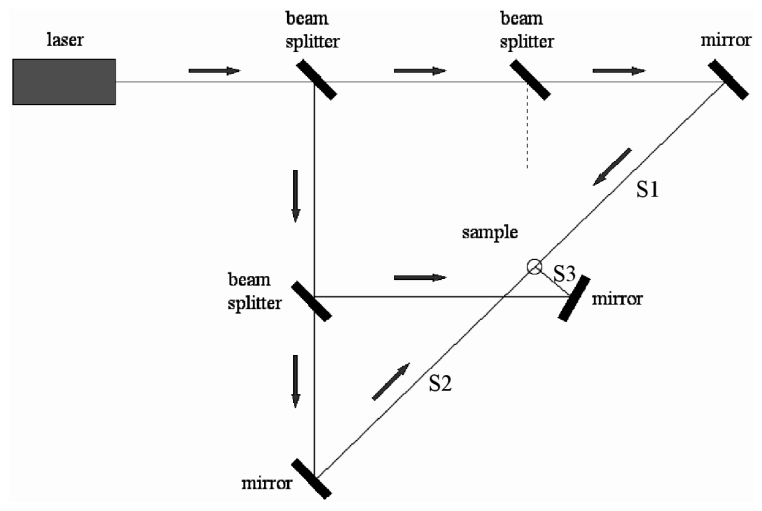

Fig. 3. Schematic representation of three beams interfering on the sample plane (top view). The sample plane is below the source plane at the distance $z=162 \mathrm{~mm}$.

was $930 \mathrm{~mJ}$. The beam from the laser passes through a series of 50:50 beam splitters, and splitted light is directed to the sample surface by mirrors. This optical configuration results in four approximately equal-intensity beams striking the sample. The angles of incidence can be varied by changing the distances between mirrors or between sample plane and mirror plane. When the beams arrive at the sample surface, optical interference occurs, and local heating at the interference maxima can modify the local material properties or even partially ablate the sample if a high light intensity is used. The distances S1 and $\mathrm{S} 2$ were equal to $140 \mathrm{~mm}$, and $\mathrm{S} 3=30 \mathrm{~mm}$. The distance between mirror plane and the sample surface was equal to $162 \mathrm{~mm}$. In order to avoid shift in the interference pattern only one pulse of laser light was chosen in each 
experiment. The irradiation experiment was performed in air at room temperature. Here we show the test results obtained with the $\mathrm{Si} /(\mathrm{Co}(1 \mathrm{~nm}) / \mathrm{Cu}(2 \mathrm{~nm}))_{3}$ multilayers prepared by thermal evaporation in UHV conditions. For the results presented here modest light intensities were used. Although the demonstrated patterning was performed for 3 beams, the primary laser beam was split into 4 beams to prevent the same intensity of interfering beams.

The obtained periodic structure were next studied with atomic force microscope (Park Instruments) working in non-contact mode.

\section{Results and discussion}

Figure 4 shows an example of the atomic force image (AFM) of the pattern produced by laser interference patterning compared with numerical simulation of interference pattern calculated at experimental conditions. One can see good correspondence between the experimental and simulated interference image. In the picture one can

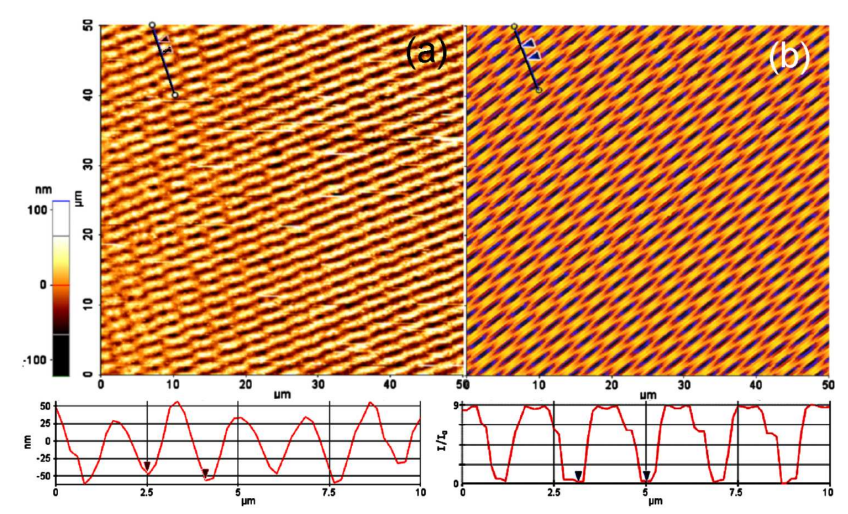

Fig. 4. AFM image of interference patterns generated by the laser beam (a) and numerical simulation calculated at the experiment conditions (b). Below the images the height profiles taken along black lines are shown. The periodicity of obtained nanostructures calculated from height profile is $1.4 \mathrm{~mm}$.

see flat islands which correspond to the destructive interference and narrow channels which correspond to the constructive interference fringes. The size of the interference pattern on the sample was about $1 \mathrm{~cm}^{2}$ and had a periodicity of $1.4 \mu \mathrm{m}$, in a reasonable agreement with the period of simulated nanostructures. Height profile curve in Fig. 4 shows the modification depth of surface reliefs along the dashed black lines drawn in Fig. 4.

\section{Conclusions}

We have simulated and experimentally investigated the formation of periodic structures generated by multibeam interference patterning. The simulations at the different setup geometry show that resulting interference pattern is quasi-periodical. On the small local areas of the sample surface the periodicity of interference pattern is preserved. For $10 \mathrm{~mm}$ laser spot diameter periodicity of the interference pattern can be assumed as constant. The interference pattern period decreases with the decrease in the laser wavelength $\lambda$, and distance $z$ between the sample plane and source plane. The simulated patterns show that the symmetries of the interference maxima depend mostly on the angles of incidence and that a wide variety of patterns can be obtained. A noticeable problem appears when one or two of the four beams are misaligned slightly out of the plane of incidence being a source of the secondary periodicity. Because of the difficulty in aligning four beams sufficiently well to avoid secondary periodicities, for testing we have used a three-beam interference configuration.

In conclusion, we have reported preliminary results concerning the use of laser beam in an interference patterning process. We have demonstrated that direct laser interference lithography can be used to prepare patterned structures on a submicron scale without need of using wet chemistry. We plan to exploit this technique further to fabricate $2 \mathrm{D}$ nanostructures of $\mathrm{FePd}$ alloy with perpendicular magnetic anisotropy as well as to use square arrays of semiconducting substrates as templates for the growth of two-dimensional structures.

\section{Acknowledgments}

This work was carried out within the framework of Polish Scientific Network MANAR.

\section{References}

[1] M. Albrecht, G. Hu, I.L. Guhr, T.C. Ulbrich, J. Boneberg, P. Leiderer, G. Schatz, Nature Mater. 4, 203 (2005).

[2] D. Makarov, L. Baraban, I.L. Guhr, J. Boneberg, H. Schift, J. Gobrecht, G. Schatz, P. Leiderer, M. Albrecht, Appl. Phys. Lett. 90, 093117 (2007).

[3] T.A. George, Z. Li, M. Yan, Y. Xu, R. Skomski, D.J. Sellmyer, J. Appl. Phys. 103, 07D502 (2008).

[4] M. Zheng, M. Yu, Y. Liu, R. Skomski, S.H. Liou, D.J. Sellmyer, V.N. Petryakov, Yu.K. Verevkin, N.I. Polushkin, N.N. Salashchenko, Appl. Phys. Lett. 79, 2606 (2001).

[5] A. Lasagni, C. Holzapfel, F. Mücklich, Adv. Eng. Mater. 7, 487 (2005).

[6] S.M. Metev, V.P. Veiko, Laser-Assisted Micro-Technology, 2nd ed., Springer, Berlin 1998. 